\title{
ROBUST DATA DETECTION IN ASYNCHRONOUS DS-CDMA IN THE PRESENCE OF TIMING UNCERTAINTY
}

\author{
Jaume Riba, Jason Goldberg, and Gregori Vázquez \\ Department of Signal Theory and Communications, E.T.S.E. Telecomunicació, Campus Nord UPC. \\ Edifici D-5, c/. Gran Capità. s/n, 08034 Barcelona, Spain, Email: \{jriba,jason,gregori\}@gps.tsc.upc.es
}

\begin{abstract}
The decorrelating and minimum mean squared error data detectors for direct sequence code division multiple access (DS-CDMA) communications systems are known to exhibit low vulnerability to the near-far problem. Nevertheless, the performance of these algorithms is highly sensitive to accurate knowledge of the user propagation delays as well as inter-symbol and/or inter-chip interference such as that produced by frequency-selective fading channels. In this paper, a new sub-optimum symbol-by-symbol detector is presented which is robust in the presence of these two effects.
\end{abstract}

\section{INTRODUCTION}

Direct-sequence code division multiple access (DS-CDMA) communications systems have recently received increased attention as a promising candidate for emerging mobile digital radio networks. For this reason, much work has been reported on the problem of multi-user detection in DS-CDMA. For asynchronous systems, the standard matched filter-bank detector is known to fail for users of widely disparate power (the so-called "near-far problem"). Moreover, the unrealistically high computational complexity of the optimum (i.e., minimum probability of error) detector [1] has motivated research on sub-optimum multi-user detectors [2]-[3].

Such systems rely on exact knowledge of additional parameters such as carrier phase, signal strength, and propagation delay for each user. However, such receivers can exhibit high sensitivity to errors in estimates of these parameters, especially propagation delay, as was shown to be the case for the decorrelating detector in [4]. Similar problems would be observed for frequency-selective fading channels. This issue has been addressed in [5] where a detection scheme relying on a multipath ray model was proposed. The technique requires estimation of the propagation delays of the individual rays of each user, and data detection is subsequently performed by forming a linear combination of symbol estimates associated with each ray.

This paper presents a simple, direct approach to robust data detection in the presence of uncertain propagation delay estimates and/or frequency selective fading. The technique is based on a generalization of a maximum signal to interference plus noise ratio (MSINR) symbol-bysymbol detector (the generalization to a block approach is straightforward). The multipath/propagation time uncertainty is taken into account by a simple statistical model. The resulting detectors are shown to be near-far resistant

This work has been supported in part by EEC Contract HCM/CHRXCT-930405, PRONTIC/CICYT TIC95-1022-C0501 and CIRIT/Generalitat de Catalunya GRQ93-3021. and insensitive to "small" errors in propagation delay estimation and/or frequency selective multipath channels with "small" delay spread. The technique may also be useful in cases where accurate timing estimates are available but can be updated with relatively low frequency. In this case, the receiver is robust is the presence of small changes in timing which take place over the propagation time up-date interval. As with other sub-optimum approaches computational complexity is linear in the number of users.

\section{PROBLEM FORMULATION}

Consider a $K$ user asynchronous DS-CDMA system nominally operating over a channel with additive white Gaussian noise (AWGN). Binary Phase Shift Keying (BPSK) modulation is used. Using the notation of $[4,5]$, the symbol interval will be denoted as $T$ and the chip interval as $T_{c}=T / N$, where $N$ is the number of chips per symbol. The $k$ th user's code waveform is of unit amplitude and is denoted by $b_{k}(t)$. It can be expressed as a pulse amplitude modulation of $\left\{c_{k}(n)\right\}_{n=0}^{N-1}$, the pseudo-noise (PN) sequence associated with the $k$ th user:

$$
b_{k}(t)=\sum_{n=0}^{N-1} c_{k}(n) p\left(t-n T_{c}\right)
$$

where $p(t)$ is a pulse whose duration, in general, exceeds the chip interval, $T_{c}$. The data sequence for the $k$ th user, $d_{k}(m) \in\{-1,+1\}$, is pulse amplitude modulated by a single period of the corresponding code waveform resulting in a baseband signal written as:

$$
s_{k}(t)=\sum_{m=-\infty}^{\infty} d_{k}(m) b_{k}(t-m T)
$$

The transmitted signal is the product of the baseband signal and the carrier: $\sqrt{2 \gamma_{k}} \cos \left(\omega_{c} t+\theta_{k}^{\prime}\right)$ where $\omega_{c}, \gamma_{k}$, and $\theta_{k}^{\prime}$ respectively denote carrier frequency, $k$ th user power and carrier phase.

In general, the channel associated with the $k$ th user can be modeled as a linear time-varying system $h_{k}(t, \tau)$ which denotes the channel response at time $t$ to an impulse applied $\tau$ seconds in the past. The received signal is:

$$
\begin{aligned}
& r^{\prime}(t)=n^{\prime}(t)+ \\
& \sum_{k=1}^{K} \operatorname{Re}\left\{\sqrt{2 \gamma_{k}} \int_{-\infty}^{\infty} h_{k}(t, \tau) s_{k}(t-\tau) e^{j\left(\omega_{c} t-\omega_{c} \tau+\theta_{k}^{\prime}\right)} d \tau\right\}
\end{aligned}
$$

where $n^{\prime}(t)$ is AWGN of two-sided power spectral density level, $N_{o} / 2$. The equivalent complex baseband representa- 
tion of the signal is given as:

$r(t)=n(t)+\sum_{k=1}^{K} \sqrt{2 \gamma_{k}} \int_{-\infty}^{\infty} h_{k}(t, \tau) s_{k}(t-\tau) e^{j\left(-\omega_{c} \tau+\theta_{k}^{\prime}\right)} d \tau$

If the channel is modeled as a simple constant delay (possibly not precisely known) then its impulse response can be written as $h_{k}(t, \tau)=\delta\left(t-\tau_{k}\right)$ where it will be assumed that $\tau_{k} \in[-T / 2, T / 2)$. In this case the received signal is:

$$
r(t)=n(t)+\sum_{k=1}^{K} \sqrt{2 \gamma_{k}} e^{j \theta_{k}} s_{k}\left(t-\tau_{k}\right)
$$

where $\theta_{k}=-\omega_{c} \tau_{k}+\theta_{k}^{\prime}$. It is this model that will be used throughout the paper.

Next define the received signal vector as $\mathbf{r}(m) \in \mathbb{C}^{Q N}$ as the sum of a signal vector $\mathbf{s}(m)$ and noise vector $\mathbf{n}(m)$ :

$$
\begin{aligned}
\mathbf{r}(m) & =\left[r(m T)_{h} r\left(m T+\frac{T}{Q N}\right), \cdots, r\left([m+1] T-\frac{T}{Q N}\right)\right]^{T} \\
& =\mathbf{s}(m)+\mathbf{n}(m)
\end{aligned}
$$

where $(.)^{T}$ denotes transpose. It is not difficult to verify that the signal vector can be expressed as:

$$
\begin{aligned}
\mathbf{s}(m) & =\mathbf{J} \sum_{k=1}^{K} \sum_{i=-1}^{1} \mathbf{b}_{k}\left(\tau_{k}+i T\right) d_{k}(m+i) \\
\mathbf{J} & =\left[\mathbf{0}_{Q N}, \mathbf{I}_{Q N}, \mathbf{0}_{Q N}\right] \in \mathbb{R}^{Q N \times 3 Q N} \\
{\left[\mathbf{b}_{k}\left(\tau_{k}+i T\right)\right]_{n} } & =\sqrt{2} b_{k}\left([n-1] T / Q N-[i+1] T-\tau_{k}\right)(9) \\
n & \in\{1,2, \cdots, 3 Q N\}, i \in\{-1,0,1\}
\end{aligned}
$$

where the subscripts on the square zero and identity matrices in (8) denote the dimension of these matrices, and $[\cdot]_{n}$ denotes the $n$th element of a vector. The above expression can be written more compactly in matrix form:

$$
\begin{aligned}
\mathbf{s}(m) & =\mathbf{J} \sum_{i=-1}^{\mathbf{1}} \mathbf{B}(\tau+i \mathbf{T}) \mathbf{d}(m+i) \\
\mathbf{B}(\tau+i \mathbf{T}) & =\left[\mathbf{b}_{1}\left(\tau_{1}+i T\right), \cdots, \mathbf{b}_{K}\left(\tau_{K}+i T\right)\right] \\
\mathbf{d}(m+i) & =\left[\sqrt{\gamma_{1}} e^{j \theta_{1}} d_{1}(m+i), \cdots, \sqrt{\gamma_{K}} e^{j \theta_{K}} d_{K}(m+i)\right]^{T} \\
\tau & =\left[\tau_{1}, \cdots, \tau_{K}\right], \quad \mathbf{T}=T \underbrace{[1, \cdots, 1]}_{K \text { elements }}
\end{aligned}
$$

The correlation matrix of the received vector is:

$$
\begin{aligned}
\mathbf{R} & =E\left[\mathbf{r}(m) \mathbf{r}^{H}(m)\right] \\
& =\sum_{i=-1}^{1} \mathbf{J B}(\tau+i \mathbf{T}) \Gamma \mathbf{B}^{H}(\tau+i \mathbf{T}) \mathbf{J}^{H}+\sigma^{2} \mathbf{I} \\
\Gamma & =E\left[\mathbf{d}(m+i) \mathbf{d}^{H}(m+i)\right]
\end{aligned}
$$

where $E[\cdot]$ is the expectation operator. It is assumed that each user's symbols are uncorrelated with those of other users (implying that $\boldsymbol{\Gamma}$ is diagonal with diagonal elements equal to user powers).
Let us define the signal-to-interference-and-noise-ratio (SINR) and the mean-squared-error (MSE) as:

$$
\begin{aligned}
\operatorname{SINR}\left(\mathbf{w}_{k}\right) & =\frac{\mathbf{w}_{k}^{H} \mathbf{R}_{s_{k}} \mathbf{w}_{k}}{\mathbf{w}_{k}^{H} \mathbf{R}_{[i+n]_{k}} \mathbf{w}_{k}} \\
\operatorname{MSE}\left(\mathbf{w}_{k}\right) & =E\left[\left|d_{k}(m)-\mathbf{w}_{k}^{H} \mathbf{r}(m)\right|^{2}\right]
\end{aligned}
$$

The "signal" correlation matrix $\mathbf{R}_{s_{k}}$ is defined as the correlation matrix of the signal vector in the presence only of the $m$ th symbol of the $k$ th user:

$$
\mathbf{R}_{s_{k}}=\gamma_{k} \mathbf{J b}_{k}(0) \mathbf{b}_{k}^{H}(0) \mathbf{J}^{H}
$$

where it is noted that, without loss of generality, the associated propagation delay is set to zero: $\tau_{k}=0$. Conversely, the interference-plus-noise correlation matrix $\mathbf{R}_{[i+n]_{k}}$ is defined as the correlation matrix of the received vector in the presence only of the noise, the remaining $K-1$ users and the $(m-1)$ th and $(m+1)$ th symbols of the $k$ th user:

$$
\mathbf{R}_{[i+n]_{k}}=\mathbf{R}-\mathbf{R}_{s_{k}} .
$$

Consider now the maximum SINR (MSINR) symbol-bysymbol receiver for user $k$. It is well known that this problem can be solved using generalized eigenanalysis. The general solution of the MSINR receiver can then be written as:

$$
\begin{aligned}
\mathbf{w}_{o_{k}(M S I N R)} & =\arg \max _{\mathbf{w}_{k} \in \mathbb{C} Q N} \operatorname{SINR}\left(\mathbf{w}_{k}\right) \\
& =\alpha \mathbf{e}_{\max }\left[\mathbf{R}_{s_{k}}, \mathbf{R}_{[i+n]_{k}}\right]
\end{aligned}
$$

where $\mathbf{e}_{\max }$ denotes the generalized eigenvector associated with the maximum eigenvalue of the above matrix pencil, and $\alpha$ is an arbitrary (non-zero) constant. We can also consider a minimum mean squared error (MMSE) symbolby-symbol receiver for user $k$ similar to [6]:

$$
\begin{aligned}
\mathbf{w}_{o_{k}(M M S E)} & =\arg \min _{\mathbf{w}_{k} \in \mathbb{C} Q N} M S E\left(\mathbf{w}_{k}\right) \\
& =\beta \mathbf{R}^{-1} \mathbf{p}_{k} \quad \mathbf{p}_{k}=E\left[\mathbf{r}(m) d_{k}^{*}(m)\right]
\end{aligned}
$$

where $\beta$ is a constant. In the particular case of a rank-one matrix $\mathbf{R}_{s_{k}}$, it is well known $\mathbf{w}_{o_{k}(M S I N R)}=\mathbf{w}_{o_{k}(M M S E)}$. It is also well known that if the noise power $\sigma^{2}$ is very low compared to powers of the interferering users, the MMSE receiver acts as a decorrelator completely nulling the effect of the interferering users. That is to say, the magnitude of receiver output for the $k$ th user will be approximately zero for each of the interfering users at the times for which the output provides an estimate for the $k$ th user's symbols. However, under these low noise conditions, it has recently been shown that inaccurate timing estimates for the users can drastically reduce performance resulting in high receiver sensitivity to near-far effects [4]. The problem addressed in this paper is the design of near-far resistant receivers that are robust in the presence of such timing errors.

\section{EFFECT OF TIMING ERRORS}

In practice, since $\tau$ is not directly available, estimates of the propagation delays, $\hat{\tau}=\left[\hat{\tau}_{1}, \cdots, \hat{\tau}_{K}\right]^{T}$, are used in the design 
of the receivers. In this section, the effects of timing error on output SINR and MSE as defined in (13) are quantified.

It will be assumed that the timing estimates, $\hat{\tau}_{k}$, can be expressed as a sum of the true propagation delay, $\tau_{k}$, and a zero mean Gaussian random variable, $\epsilon_{k}$ of variance $\sigma_{\epsilon_{k}}^{2}$.

$$
\hat{\tau}_{k}=\tau_{k}+\epsilon_{k}, \quad E_{\epsilon}\left[\epsilon_{k}\right]=0, \quad E_{\epsilon}\left[\epsilon_{k} \epsilon_{k^{\prime}}\right]=\sigma_{\epsilon_{k}}^{2} \delta\left(k-k^{\prime}\right)
$$

where $E_{\epsilon}$ is the expectation over the $K$ propagation delay errors. The above model also accounts for the multipath effects appearing in frequency selective fading channels (with small delay-spread). Then, in presence of timing errors, a corresponding average output SINR can be defined as:

$$
\begin{aligned}
\overline{\operatorname{SINR}}\left(\mathbf{w}_{k}\right) & =\frac{\mathbf{w}_{k}^{H} \overline{\mathbf{R}}_{s_{k}} \mathbf{w}_{k}}{\mathbf{w}_{k}^{H} \overline{\mathbf{R}}_{[i+n]_{k}} \mathbf{w}_{k}} \\
\overline{\mathbf{R}}_{s_{k}} & =E_{\epsilon}\left[\mathbf{R}_{s_{k}}\right] \quad \overline{\mathbf{R}}_{[i+n]_{k}}=E_{\epsilon}\left[\mathbf{R}_{[i+n]_{k}}\right]
\end{aligned}
$$

Similarly, a corresponding average MSE can be defined as:

$$
\overline{\operatorname{MSE}}\left(\mathbf{w}_{k}\right)=E_{\epsilon} E\left[\left|d_{k}(m)-\mathbf{w}_{k}^{H} \mathbf{r}(m)\right|^{2}\right]
$$

We propose the definitions (19) and (21) as measures of performance in presence of timing uncertainty.

\section{ROBUST DETECTOR}

In the absence of timing errors, the decorrelating detector (and the MMSE detector as $\sigma^{2} \rightarrow 0$ ) completely null out the influence of all interfering signature waveforms at their specified timings (as defined by their propagation delays). In the presence of timing errors, such nulling is not guaranteed. Even for small errors the reduction in performance as measured by decrease in average SINR (19) or by increase in average MMSE (21) can be very significant, especially for high near-far scenarios. The effect of the timing errors as seen by averaging over the propagation delays corresponds to a sort of temporal smearing of each of the $K$ user's signals. A detector which is designed to be robust in the presence of timing errors should take this smearing effect into account in order to create broad temporal nulls for the interfering users. To this end, we can define the robust MSINR and robust MMSE receivers as:

$$
\begin{aligned}
\mathbf{w}_{r_{k}(M S I N R)} & =\arg \max _{\mathbf{w}_{k} \in \mathbb{C} Q N} \overline{\operatorname{SINR}}\left(\mathbf{w}_{k}\right) \\
& =\mathbf{e}_{\max }\left[\overline{\mathbf{R}}_{s_{k}}, \overline{\mathbf{R}}_{[i+n]_{k}}\right] \\
\mathbf{w}_{r_{k}(M M S E)} & =\arg \min _{\mathbf{w}_{k} \in \mathbb{C} Q N} \overline{\operatorname{MSE}}\left(\mathbf{w}_{k}\right) \\
& =\beta \overline{\mathbf{R}}^{-1} \overline{\mathbf{p}}_{k} \quad \overline{\mathbf{p}}_{k}=E_{\epsilon} E\left[\mathbf{r}(m) d_{k}^{*}(m)\right]
\end{aligned}
$$

Due to the expectation over the $k$ th propagation delay error, $\epsilon_{k}$, the "signal" correlation matrix, $\overline{\mathbf{R}}_{s_{k}}$, has lost the rank-one property and, therefore, the two solutions are not equal in this case: $\mathbf{w}_{r_{k}(M S I N R)} \neq \mathbf{w}_{r_{k} \text { (MMSE) }}$.

Finally, transformation to the Fourier domain, where a time-shift corresponds to a linear phase, will aid in formulation of simple closed form expressions for the average correlation matrices (20). In particular, we define a Discrete
Fourier Transform (DFT) matrix as:

$$
\begin{aligned}
\mathbf{F} & =\frac{1}{\sqrt{3 \overline{Q N}}}\left[\begin{array}{cccc}
1 & e^{j 2 \pi \frac{M}{3 Q N}} & \cdots & e^{j 2 \pi \frac{M(3 Q N-1)}{3 Q N}} \\
1 & e^{j 2 \pi \frac{M-1}{3 Q N}} & \cdots & e^{j 2 \pi \frac{(M-1)(3 Q N-1)}{3 Q N}} \\
\vdots & \vdots & \ddots & \vdots \\
1 & e^{-j 2 \pi \frac{M}{3 Q N}} & \cdots & e^{-j 2 \pi \frac{M(3 Q N-1)}{3 Q N}}
\end{array}\right] \\
M & =(3 Q N-1) / 2 .
\end{aligned}
$$

Now, resorting to the time-shift property, the DFT of the users' code waveforms can be writen as:

$$
\begin{aligned}
\mathbf{A}(\tau+\epsilon) & =\mathbf{F B}(\tau+\epsilon)=\mathbf{A}(\tau) \odot \mathbf{V}(\epsilon) \\
\mathbf{A}(\tau) & =\mathbf{A}(0) \odot \mathbf{V}(\tau)
\end{aligned}
$$

where $\odot$ denotes the element-wise Schur product, and the linear-phase matrix $\mathbf{V}(\mathbf{x})$ and vector $\mathbf{v}(x)$ are defined respectively as:

$$
\begin{aligned}
& \mathbf{v}(\mathbf{x})=\left[\mathbf{v}\left(x_{1}\right), \mathbf{v}\left(x_{2}\right), \cdots, \mathbf{v}\left(x_{K}\right)\right] \\
& \mathbf{v}(x)=\left[e^{-j 2 \pi M x / 3 T}, e^{-j 2 \pi(M-1) x / 3 T}, \cdots, e^{j 2 \pi M x / 3 T}\right]^{H}
\end{aligned}
$$

The unitary property of $\mathrm{F}$ and the linearity of the expectation operator imply:

$$
\begin{aligned}
\overline{\mathbf{R}} & =\mathbf{J} \mathbf{F}^{H}\left[\left(\mathbf{A}(\tau) \Gamma \mathbf{A}^{H}(\tau)\right) \odot \mathbf{S} \odot \mathbf{Q}\left(\sigma_{\epsilon_{\max }}^{2}\right)\right] \mathbf{F} \mathbf{J}^{H}+\sigma^{2} \mathbf{I} \\
\mathbf{S} & =\sum_{i=-1}^{1} \mathbf{v}(i T) \mathbf{v}^{H}(i T) \\
\overline{\mathbf{R}}_{s_{k}} & =\gamma_{k} \mathbf{J} \mathbf{F}^{H}\left[\left(\mathbf{a}_{k}(0) \mathbf{a}_{k}^{H}(0)\right) \odot \mathbf{Q}\left(\sigma_{\epsilon_{\max }}^{2}\right)\right] \mathbf{F J} \\
\overline{\mathbf{R}}_{[i+n]_{k}} & =\overline{\mathbf{R}}-\overline{\mathbf{R}}_{s_{k}}
\end{aligned}
$$

where as in [7]:

$$
\left[\mathbf{Q}\left(\sigma_{\epsilon_{\max }}^{2}\right)\right]_{p q}=\left[E_{\epsilon}\left[\mathbf{v}(\varepsilon) \mathbf{v}^{H}(\varepsilon)\right]\right]_{p q}=e^{-2 \sigma_{\epsilon \max }^{2}[\pi(p-q)]^{2}}
$$

The problem of the exact propagation delays appearing in the parameterized computation of $\overline{\mathbf{R}}$ in (26) can be addressed by simply using the estimated delays, $\hat{\tau}_{k}$, in the arguments of the $\mathbf{V}(\cdot)$ and $\mathbf{v}(\cdot)$ in the computation of $\mathbf{A}(\tau)$ using (24) and (25).

Thus, in summary, the new robust MSINR receiver filter is formed by using (27) and (28) with estimated propagation delays in (22). The robust receiver (22) offers, as verified in the next section, the following compromise with respect to the optimum receiver defined in (16):

$$
\operatorname{SINR}\left(\mathbf{w}_{o_{k}}\right)>\operatorname{SINR}\left(\mathbf{w}_{r_{k}}\right) \approx \overline{\operatorname{SINR}}\left(\mathbf{w}_{r_{k}}\right)>>\overline{\operatorname{SINR}}\left(\mathbf{w}_{o_{k}}\right)
$$

\section{RESULTS}

In this section, results of computer simulations of the performance of the new technique (22) and comparison with that of the ordinary MMSE/MSINR receiver (17) are presented. Consider a $K=3$ user with $Q=2$ samples per chip and $N=31$ chips per symbol. Nyquist pulses with roll-off 0.5 are used, and, for convenience $T=1$. The performance of receiver's for user $k=1$ are consider with $\tau_{1}=0$, $\tau_{2}=T / 3$, and $\tau_{3}=-T / 3$ and user powers $\gamma_{1}=1, \gamma_{2}=50$, 
and $\gamma_{3}=50$. To gain insight into the effect of the new procedure, consider Fig. 1, the output of the conventional receiver (designed for the above scenario) for user one when only one symbol of user two is present at its input. The receiver succeds in placing two sharp null at times $t=0$ and $t=T$ over the interfering user. Now, consider the same experiment but with the robust receiver of (22) as shown in Fig. 2. This time two broad temporal nulls are placed at times $t=0$ and $t=T$. These broad nulls are what provides the robustness to timing uncertainty.

Next, for the above user powers and noise power $\sigma^{2}=$ 0.1 , Fig. 3 shows the output SINR as defined in (19) for the ordinary receiver (assumed timing error variance zero) and the robust receiver (assumed timing error variance, $\sigma_{\epsilon_{\max }}^{2}=0.003$ ) as the true timing error variance is varied. The curves indicate that, even for relatively low nearfar and low timing error variance, the conventional receiver is highly sensitive to uncertainty in the propagation delays while the robust receiver offers nearly constant performance with timing error variance. Lastly, Fig. 4 shows performance for the conventional and robust receivers as a function of the ratio of interferer power, $\gamma_{2}=\gamma_{3}$, to desired user power, $\gamma_{1}$. The true timing error variance as well as that used in the design of the the robust receiver are again set to $\sigma_{\varepsilon_{\max }}^{2}=0.003$. The conventional receiver is far more sensitive to interferer power than the robust technique.

\section{CONCLUSIONS}

A new method for the design of multi-user detectors which are robust in the presence of propagation time estimation errors and/or frequency selective multipath (with delay spread on the order of a few chips) has been presented. The new detector is near-far resistant and offers greatly improved performance over the conventional detector for a variety of scenarios. The technique is also useful for robust detection in cases where highly accurate timing estimates are available but can only be updated relatively infrequently.

\section{REFERENCES}

[1] S. Verdú. "Minimum probability of error for asynchronous Gaussian multiple access channels". IEEE Transactions on Information Theory, vol. 32: pp. 8596, January 1986.

[2] R. Lupas and S. Verdú. "Near-far resistance of multiuser detectors in asynchronous channels". IEEE Transactions on Communications, vol. 38: pp. 496-508, April 1986.

[3] Z. Xie, R.T. Short, and C.K. Rushforth. "A family of suboptimum detectors for coherent multiuser communication". IEEE Journal on Selected Areas in Communications, vol. 8: pp. 683-690, May 1990.

[4] S. Parkvall, E. Ström, and B. Ottersten. "The impact of timing errors on the performance of linear DSCDMA receivers". submitted to IEEE Journal on Selected Areas in Communications, April 1995.

[5] S. Parkvall and E. Ström. Parameter estimation and detection of DS-CDMA signals subject to multipath propagation. In Proceedings of IEEE/IEE Workshop on Signal Processing in Multipath Environments, Glasgow, Scotland. IEEE/IEE, 1995.

[6] E. Ström and S. Parkvall. Joint parameter estimation and detection DS-CDMA signals in fading channels. In
Proceedings of IEEE Global Telecommunications Conference, 1995.

[7] T. Trump and B. Ottersten. "Estimation of nominal direction of arrival and angular spread using an array of sensors". submitted to Signal Processing, 1994.

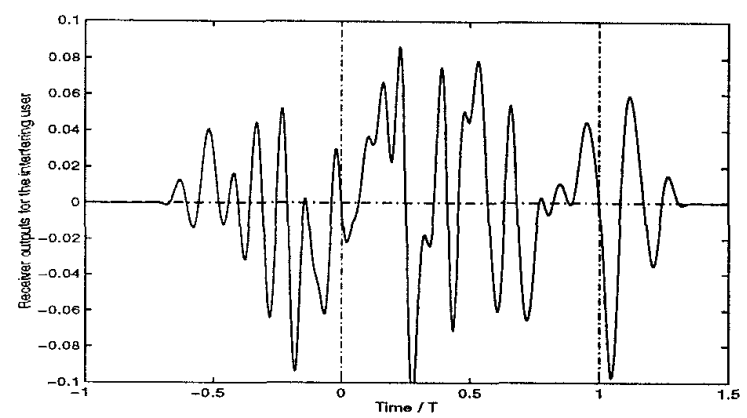

Figure 1: Conventional receiver one output vs. time

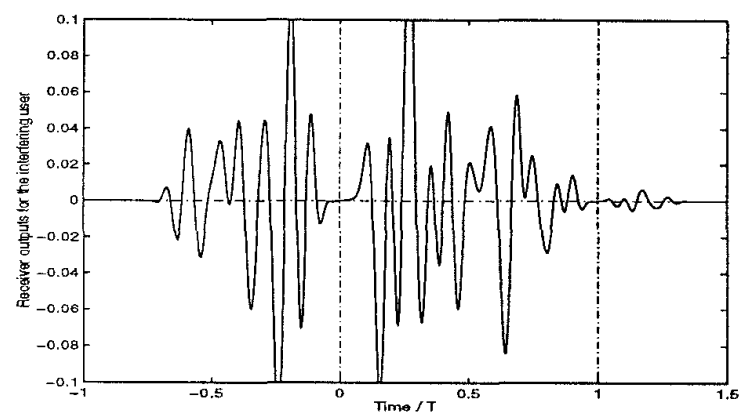

Figure 2: Robust receiver one output vs. time

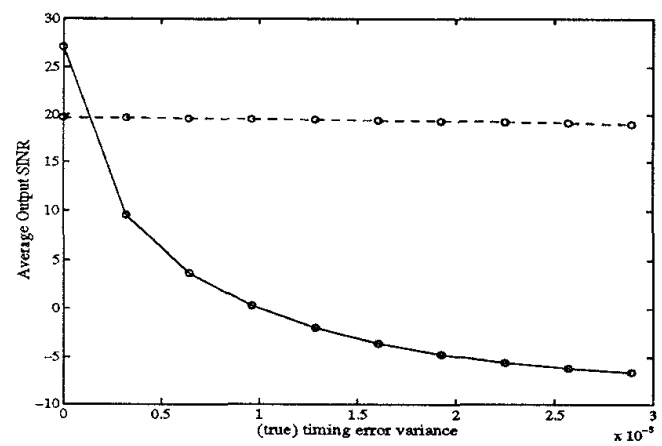

Figure 3: SINR vs. timing error var. (- conv., - - rob.)

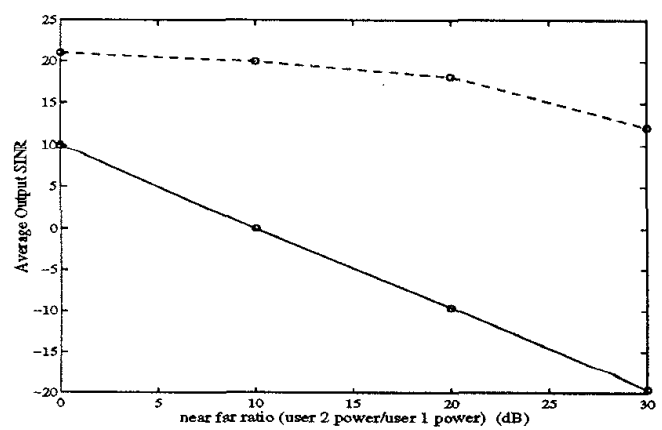

Figure 4: SINR vs. $\gamma_{2} / \gamma_{1}$ (- conv., - - rob.) 